\title{
Toward Agile Strategies for Enhancing Community Resilience Following the COVID-19 Pandemic: An Interview Study
}

\section{Hossein Mokhtarzadeh}

The global Pandemic as a result of a recently discovered coronavirus has affected every aspect of our lives. In this observational study, I interviewed a small group of experts in different fields on how they cope with this global crisis. Have they modified their strategies to achieve their goals and whether this has affected them negatively or positively? The interviewees were from academia, and industry. Almost all participants found the lockdowns quite positive, however, a sentiment analysis revealed a negative outcome with moderate confidence (54.3\%) with Australian participants; and positive (96.2\% confidence) when all participants were included. Some of the positive outcomes included more time with family, more physical activities, and creative ways to perform tasks. Negative outcomes involved some of their team members who could not handle the new norms. Further research is required to be conducted with a wider range of stakeholders to better understand how we can recover more efficiency from this pandemic in Australia and beyond.

\subsection{Introduction}

Global pandemic was announced on 11 March 2020 by World Health Organization (WHO) as a result of COVID-19 outbreak. Since its first identification in Wuhan, China, in December 2019, these tiny (i.e. $65-125 \mathrm{~nm}$ ) coronaviruses [1] traveled by humans across the globe in a short time thus changed our lives for good. Since its inception, COVID-19 disease has taken over 875K lives and infected 26.6M worldwide at the time of writing (on 6 September 2020) [2]. Finding a cure or effective treatment for COVID-19 is yet to be identified. As a result of its devastating consequences [3], governments have implemented serious measures such as stay at home orders, mandatory wearing masks in public, shutting down businesses and disrupting international travels. These extraordinary steps to contain this infectious disease have led to both health and economical disasters [4]. For instance, Australia may be facing its first ever recession in the last three decades [5]. To successfully overcome the COVID-19 crisis, adaptation to new norms and agile mindsets are recommended; however, some argue that agile and adaptative governance may not always go hand in hand in crisis response [6]. These negative side effects of pandemic require optimal strategies stemming from governments and ordinary people backed by science that can be swiftly tested and readjusted. Having these challenges in mind, I turned to experts to better understand how they evaluate the situation, how they cope with the pandemic, and how they think industries and businesses can thrive following/during the pandemic. Therefore, in this observational study, I 
interviewed experts [7] from academia, industry to gain their insights regarding the consequences of the pandemic and whether they plan to modify their strategies to achieve their goals.

\subsection{Method}

I interviewed six experts in different fields from industry and academia from April to July $2020^{1}$. Originally, I prepared some predefined questions (Table 37.1); however, the discussion was open for the interviewees to share their observations during the pandemic. I did not necessarily follow the questions in Table 37.1 as the discussion progressed. The main aim was to ask them whether they have changed their strategies following pandemic.

I produced word clouds of transcripts of all interviews. I first collected transcripts of all interviews from YouTube's free transcription tool embedded in each video. Then, a sentiment analysis was performed on the whole interviews using a simple online tool from https:// monkeylearn.com/. Sentiment categories of positive, negative and neutral were done automatically on the transcripts of interviews. Finally, top 20 keywords from the interviews were extracted from a keyword extractor tool on MonkeyLearn website.

\section{TABLE 37.1}

Some predefined questions to ask participants during interviews. Not all the questions were asked during interviews.

\begin{tabular}{ll}
\hline$\#$ & Questions \\
\hline 1 & $\begin{array}{l}\text { How pandemic has changed your strategies in your own career and } \\
\text { company? }\end{array}$ \\
\hline 2 & $\begin{array}{l}\text { Are you still adjusting to new ways of working (e.g. WFH, } \\
\text { collaboration, etc.)? If yes, why and how? }\end{array}$ \\
\hline 3 & What is your highlight of this period for you? \\
\hline 4 & $\begin{array}{l}\text { What are you learning now that you may apply in other unexpected } \\
\text { events in the future? }\end{array}$ \\
\hline 5 & $\begin{array}{l}\text { What are our advantages/disadvantages in Australia regarding } \\
\text { building industries considering pandemic? }\end{array}$ \\
\hline 6 & $\begin{array}{l}\text { Who would you like to hear about their strategy these days or as } \\
\text { your role model? }\end{array}$ \\
\hline
\end{tabular}

\subsection{Results}

Interviews took over 3 hours with a mean and standard deviation of $32 \pm 5$ min for each. I observed that the interviewee's description of events were quite positive; however, using sentiment analysis, it was categorized "negative" (54.3\% confidence) when all Australian participants were involved. Nevertheless, the sentiment analysis presented positive (96.2\% confidence) when all participants' data was analyzed.

Among the top 20 keywords extracted from the transcripts using an online keyword extractor were the following words: "people"(197 times), time (81 times), "jobs" (64 times), "industry" (56 times), "strategy" (55 times), and "pandemic" (39 times).

Almost all participants were fine by working from home (WFH) which has also been recently confirmed in a large scale study in the US [8]. Academics in this study agreed that future directions of education could be hybrid combining face to face and in person trainings. CEOs agree that most

\footnotetext{
${ }^{1}$ All these interviews and their details are freely available on YouTube (shorturl.at/clsxH).
} 
senior managers are quite busy during pandemic and consider it as a new opportunity even if one needs to look a bit harder to find them.

\subsection{Discussion and Conclusion}

In this observational study, I interviewed six experts in different fields regarding their professional and personal strategies during the pandemic. The interviewees agreed that the pandemic provides new opportunities despite health and economic challenges. For instance, these opportunities included being with family as a result of working from home and finding creative ways to adjust the business and return of cashflow during the pandemic. However, given a small sample size, I found that certain occupations (e.g. human performance and sense of balance) which deal with mind-body illustrated more positive (high confidence) category compared to interviewees from Australia when transcriptions were analyzed using sentiment analysis. These differences could be related to the type of questions I asked during the interviews and cannot be generalized. The pandemic arguably challenged most of our established methods (or strategies) of performing tasks. Work from home, online education, telemedicine and even online job interviews are widespread and acceptable, which may even be a new norm post-pandemic.

The top keywords extracted from the interviews referred to "people", "time", "industry" and "jobs" which may show the extent of concern individuals would have regarding job security in a timely manner. These findings indeed were interesting since the interviews were not necessarily about the job security. Though the findings require further scrutiny, such interviews and their analyses may provide a framework to explore how individuals' strategies can lead to prosperity post-pandemic [9]. Such understanding enables us to better choose our future endeavors and the values we appreciate. Collectively, this preliminary project reveals that we can develop agile strategies in a short time and be quite adaptive.

In conclusion, this preliminary study could pave the path toward more agile mindset to optimize our strategies in achieving our goals. Such an approach can be extended to larger scale decision making processes in a city, state, country or global challenges. Moreover, future studies can take advance of qualitative methods such as Interpretative Phenomenological Analysis to identify the underlying challenges everyone undergoes during the pandemic [10]. We need to develop these methods in advance to avoid the next global crisis affecting community resilience which may appear in a form of another pandemic or other disasters such a climate change.

\section{Acknowledgement}

I would like to sincerely thank all the guests of my program on YouTube Channel (shorturl.at/ $\mathrm{clsxH})$.

\section{References}

[1] Muhammad Adnan Shereen, Suliman Khan, Abeer Kazmi, Nadia Bashir, and Rabeea Siddique. Covid-19 infection: Origin, transmission, and characteristics of human coronaviruses. Journal of Advanced Research, 2020.

[2] Wikipedia. Covid-19 pandemic data, 2020. URL https://en.wikipedia.org/wiki/Template:COVID-19_pandemic_ data. 
[3] Maria Nicola, Zaid Alsafi, Catrin Sohrabi, Ahmed Kerwan, Ahmed Al-Jabir, Christos Iosifidis, Maliha Agha, and Riaz Agha. The socio-economic implications of the coronavirus and covid-19 pandemic: a review. International Journal of Surgery, 2020.

[4] Angel Carlos Bassols and Bassols Fj. The oil industry and its relation to the pandemic covid 19. Archives of Petroleum \&S Environmental Biotechnology, 5:164, 082020.

[5] Nassim Khadem. Australians are hurting from the coronavirus-led recession, but we fare better than most countries, 2020. URL https://www.abc.net.au/news/2020-06-05/australia-coronavirus-recession-comparesinternational-countries/12322260.

[6] Marijn Janssen and Haiko van der Voort. Agile and adaptive governance in crisis response: Lessons from the covid-19 pandemic. International Journal of Information Management, page 102180, 2020.

[7] H Mokhtarzadeh. Strategy interviews [internet], 2020. URL https://www.youtube.com/channel/UCeV5XhAkh_ jcWmKylTIP38Q.

[8] E Bernstein, H Blunden, A Brodsky, W Sohn, and B Waber. The implications of working without an office, 2020. URL https://hbr.org/2020/07/the-implications-of-working-without-an-office.

[9] Ivan Hernandez, Daniel A Newman, and Gahyun Jeon. Twitter analysis: Methods for data management and a word count dictionary to measure city-level job satisfaction. 2016.

[10] Jonathan A Smith and Pnina Shinebourne. Interpretative phenomenological analysis. American Psychological Association, 2012. 